\title{
Anodic Acetoxylation of Anisole at Graphite and Platinum Electrodes
}

\author{
C.Thillaiyadi Valliammai, M.Pugazhenthi and S.Sivaji Ganesan \\ Department of Chemistry,AVVM Sri Pushpam College (Autonomous),POONDI-613503 (TN) INDIA.
}

\begin{abstract}
Anodic acetoxylation of anisole has been carried out on graphite and platinum electrodes in glacial acetic acid and sodium acetate. On graphite electrode p-acetoxy anisole is the major product along with hydroxy derivative in traces. By using platinum electrode, the major product is dimer along with hydroxy derivative in traces. The most probable mechanism has been proposed.
\end{abstract}

Keywords: Anodic acetoxylation, Anisole, Graphite and platinum electrodes.

\section{Introduction}

Anodic acetoxylation of p. methyl anisole in glacial acetic acid using graphite and boron doped diamond have been studied by Fankhauser and his Co-workers ${ }^{1}$. A number of other workers hold similar such views ${ }^{2-4}$. In the present work, anodic acetoxylation of anisole has been carried out on graphite and platinum electrodes in glacial acetic acid and sodium acetate.

\section{Materials}

\section{Experimental}

Anisole (BDH) was purified by vaccum distillation. The purity was checked by physical parameters and confirmed by TLC.

\section{Electrochemical Process}

The anodic acetoxylation reaction was carried out galvanostatically in a divided cell and locally fabricated $5 \mathrm{v}-100 \mathrm{~mA}$ regulated DC power supply. The potentials were measured as cell voltages. Current voltage studies were performed with graphite and platinum anode in glacial acetic acid and sodium acetate.

The anolyte contained $0.1 \mathrm{M}$ substrate in 5\% glacial acetic acid 20\% sodium acetate mixture. Assuming 2electron process to occur depending on the intensity of current realized at the given potential, the duration of current passed was fixed whenever current decrease was unduly large either the polarity was reversed or potential was taken to zero volt for a short period as practiced in established electro synthetic works ${ }^{5,6}$.

\section{Product Analysis}

The organic product mixture was separated by ether extraction and resolved on TLC test plate, using 10:1 benzene-ether mixture as elutent. The products were identified by means of co-TLC, physical constants, chemical analysis and pmr spectral analysis.

\section{Results And Discussion}

The current voltage curves for the systems suggests, the working potential range on graphite anode was observed to be $0.9 \mathrm{v}$ to $1.0 \mathrm{v}$. The working potential range on platinum anode was observed to be $1.4 \mathrm{v}$ to $1.85 \mathrm{v}$. By using graphite electrode, p-acetoxy anisole as the major product and hydroxy anisole in traces. On platinum electrode, dimer as the major product along with hydroxy anisole in traces.

Graphite anode facilitates adsorption controlled phenomenon, on platinum anode diffusion controlled process occurs. By using graphite or platinum loss of 1 electron occur from the system initially to form radical cation.

On graphite anode, radical cation gets chemisorbed and it is induced by nucleophilic attack of OAC ${ }^{-}$. This results in nucleophilic addition By using platinum anode different situation arises As soon as radical ion is formed it gets diffused into the bulk solution away from the electrical double layer. The labile radical ion undergo dimerisation and it might be exposed to nucleophilic attack by $\mathrm{OAC}^{-}$in the solution.

The attack of the neutral substrate molecule with its $\pi$ electron density on the cation radical to form $\pi$ complex .The $\pi$ complex thus formed may subsequently develop into $\sigma$ complex which is again a radical ion. The $\sigma$ complex further undergo second 1-electron transfer to form the dication. On deprotonation, dimers results. 
The following mechanism has been proposed for acetoxylation of anisole Formation of p-acetoxy anisole and p-hydroxy anisole

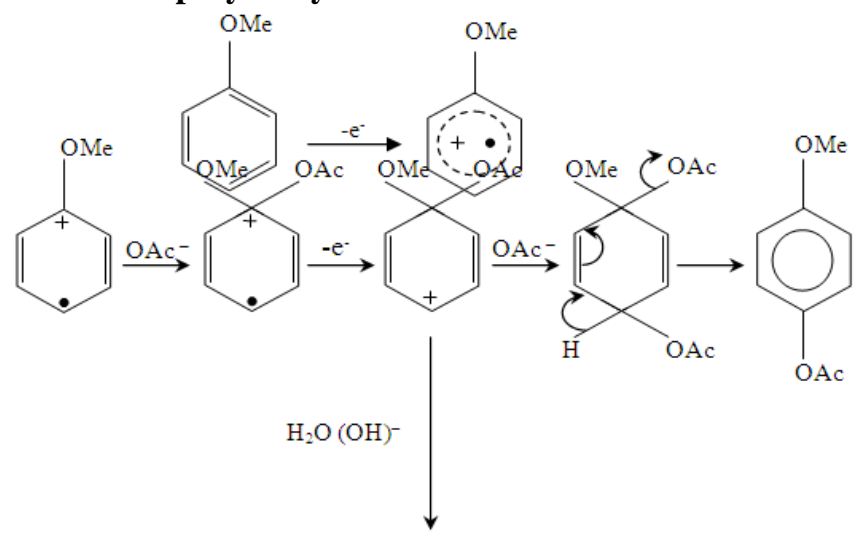

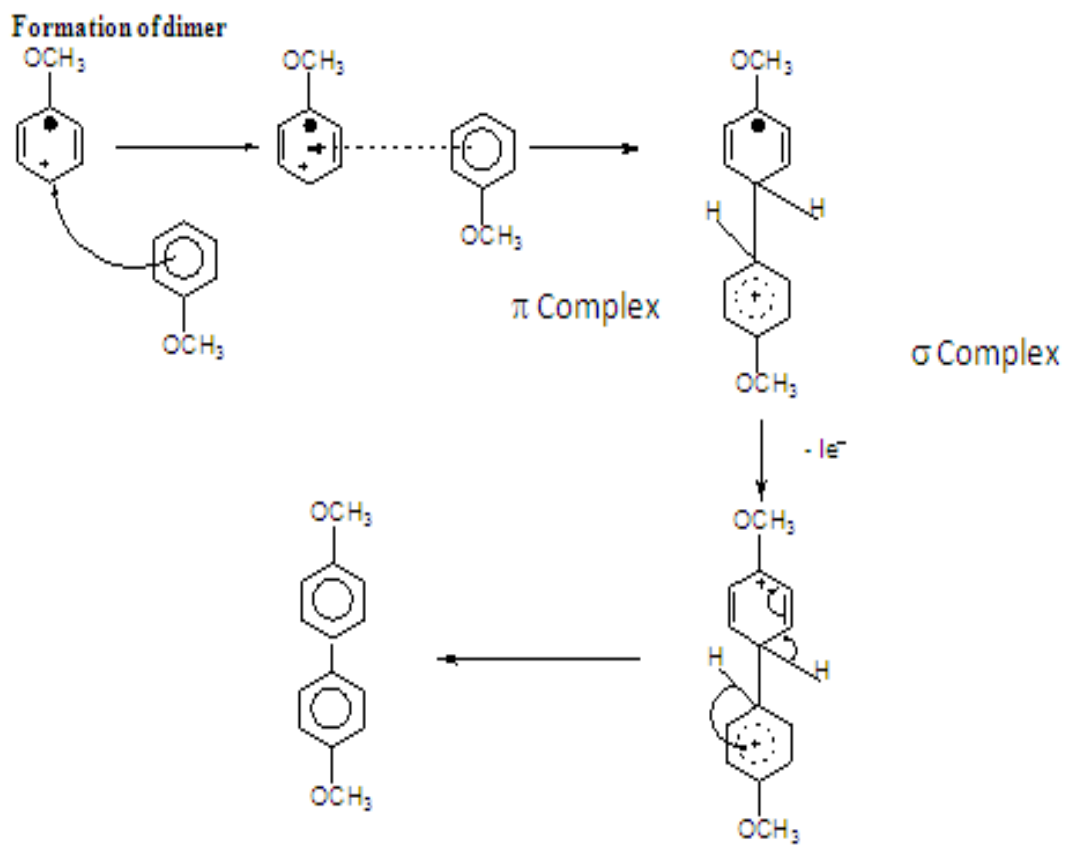

Such a multiplex approach finds references in the earlier literature. 1, 4- Dimethoxy benzene has been reported to undergo electrochemical reactions in three different pathways viz., ring substitution, ipso substitution and quinone formation ${ }^{7-10}$. On platinum the attack of the neutral substrate molecule on the cation radical to yield the dimerized product has been favorably proposed ${ }^{11-12}$.

\section{References:}

[1]. Ulrich Griesbach, Andreas Fischer, Hermann Putter and Christos Comniellin, J.Electro anal. Chem., 614 (2008) 107-112.

[2]. Kowalski.J, Ploszynska.J, Sobkowiak.A, Moraycki J.W. and Wilczewska A.Z, J. Electro anal. Chem., 4 (2005) 275-280.

[3]. Toshio Fuchigami, Yuki Nakagawa and Tsutoma Nonaka Tetrahedran 27 (1986) 3869-3872.

[4]. J.Hoffmann and A.Sarafidis, Jovoss, Electro Chemica Acta; 36 (1991) 1165-1169.

[5]. H.J.Schafer Topics in Current Chemistry; 152 (1990) 91.

[6]. Lines Robert and H.P Utley James, J.Chem Soc. Perkin Trans. II 803 (1977).

[7]. J. Wang, M. Jiang, and F. Lu, J.Electroanal. Chem.444 (1998) 127.

[8]. C.X. Zhao, Y.F. Gong, H.Y. He and X.K. Jiang, J. Phys. Org. Chem., 12, 688 (1999)

[9]. J.H. Dong, H.Y. Dou and C.X. Zhao, J. Fluorine Chem., 127, 205 (2006)

[10]. Fischer, A.; Show, Y.; and Swain, G. M., Anal. Chem., 76 (2004) 2553

[11]. Swain, G. M. In Electroanalytical Chemistry; Bard A J, Rubinstein I Ed, Marcel Dekker: New York, Vol. 22, (2003) 181

[12]. Hasegawa, M.; and Fuchigami, T., Electrochim. Acta 2004, 49, 3367. 